the atmosphere by successive diffusions, I have spent
several months in experiments with this end in view. several months in experiments with this end in view. find a suitable porous septum. and sufficiently fine grained to prevent more than a very slow nassage of air, while a considerable surface is ex-
posed to the atmosphere on one side and to a fairly good vacuum on the other. Many substances were tried. India rubber save encouraging results, but
was caoricious, and very slow. The best results have feen obtained with porous porcelain having its super tube of this kind, closed at one end, and exposing air, was connected with the apparatus, and the whol kept exhausted to a pressure of $1.3 \mathrm{~mm}$. About $19 \mathrm{c}$.
of gas was diffused per hour. After 36 hours the dif asion tube was shut off, the exhaustion continued impracticable to carry the exhaustion below six mil terfered with the action of the pump, no desicating
agent being used. As both phosphorus pentoxide and soda lime absorb the new gas, they cannot be used fo
desiccation. If the exhaustion had been carried as far as in the curve, o $p$, it is probable that a higher con-
ductivity than in that curve would have been shown i. e., a conductivity more than forty-two times that of hydrogen, or something like a hundred times that of
air. Here we have the heat conductivity of air a very low vressure, increased something like a hundred diffusion, but feel confident that successive diffusions air will afford a practicable means of obtaining the new gras in a state of approximate purity.
Now let us diseover. if possible, the meaning of th
extruordinary extraordinary heat conductivity of the new gas. heat, mean molecular velocity, and heat conductivit bon dioxide, which are the known gases represente in the curve chart. For easy comparison, I have take not only the density, but the specific heat, mean moleunity. The similarity of values in the fitth and sixth
columns, for molecular velocity and heat conductivity, is striking. On the chart, the curves for carbon monis striking. On the chart, the curves for carbon mon-
oxide, air, and carbon dioxide are evidently too high cular velocity in the fitth column but these curves represent only the last; 50 millionths of the complete
curves. By following these back to 500 millionths, which is still a rather small pressure, and taking their
values at intervals of 10 millionths from no pressure upward, we obtain as a mean of all these values for These a the fairly well with the molecular velocities. COMPARISON of aASEOUS PROPERTIES.

\begin{tabular}{|c|c|c|c|c|c|}
\hline 1 & 2 & 3 & 4 & $\mathbf{5}$ & 6 \\
\hline Gas & $\begin{array}{l}\text { Molecular } \\
\text { weight }\end{array}$ & $\begin{array}{c}\text { Density } \\
\text { D }\end{array}$ & $\begin{array}{c}\text { Relative } \\
\text { specific } \\
\text { heat }\end{array}$ & $\begin{array}{c}\text { Relative } \\
\text { molecular } \\
\text { velocity } \\
\frac{1}{\sqrt{D}}\end{array}$ & $\begin{array}{l}\text { Relative } \\
\text { heat } \\
\text { conduc- } \\
\text { livity }\end{array}$ \\
\hline Etherion & ${ }^{2} 0.0002$ & ? 0.0001 & $6000 .^{?}$ & $100{ }^{?}$ & 100. \\
\hline Hydrogen & 2. & 1. & 1. & 1. & 1. \\
\hline Helium & 4. & 2. & 800 & 0.71 & 0.78 \\
\hline $\begin{array}{l}\text { Carbon } \\
\text { nonoxide }\end{array}$ & 27.8 & 18.8 & 0.072 & 0.27 & 0.88 \\
\hline Air & 28.8 & 14.4 & 0.068 & 0.28 & 0.82 \\
\hline $\begin{array}{l}\text { Carbon } \\
\text { dioxide }\end{array}$ & 48.8 & 21.8 & 0.084 & 0.21 & 0.21 \\
\hline
\end{tabular}

I offer the suggestion that the relatively high conductivity of the last three gases at the low pressure of their molecules by unobstructedimpact on the walls and more retarded as the pressure is lessened, because the greater separation of the molecules reduces the
frequency of collisions. Helium, perhaps because it is frequency of collisions. Helium, perhaps because it is
monatomic and therefore incapable of dissociation, ratio, given in the sixth column, varies but little more than a thousand millionths. I have taken the density of helium at 2 ; but. Prof. Ramsay finds it a
little less than this when purified as far as possible by repeated diffusions. This makes its relative noolecular to the observed value of its heat conductivity.

From the foregoing, we may reasonably. that the heat conductivity of gases at low pressures, and their mean molecular veiocities, are closelv related. otherwise unknown gas, we can form some idea of it As before indicated, the heat conductivity of the new gas at very' low pressure, even when mixed with
large proportion of other gases, is something like hundred times that of hydroges. I shall not be sur-
prised to find the conductivity of the pure gas a thou-
sand or more times greater than that of hydrogen; but let us be conservative, and for the present purpose given it this value at the head of the sixth column.
If my inference that the heat conductivity and molecular velocity of gases are directly related is correct.
then the molecular velocity of the new gas will be 100 times that of hydrogen, as shown at the head of the
fifth column. As is weli known, the mean molecular of its absolute temperature, and is independent of pressure. The mean molecular velocity of hydrogen calculation to be 5,571 feet per second. Hence the
mean molerular velo' mean uolesular velority of the new gas at the same
temperature will be 557.100 feet, or more than 105 miles per second. At anything like this molecular velocity atmosphere, unless the space above also contained it
A velocity of only about seven miles per second, if un
checked except by gravitation, would be sufficient to project a body from the earth permanently into space
Even at a temperature very close to absolute zero, th
new gas would have sufficient molecular velocity to scape from the atmospher

Again, inasnuch as the molecular velocities of gases
vary in versely as the square roots of their densities, it follows from our assumption of its molecular velocity
that the density of the new gas is only the ten thou andth part that of hydrogen, as shown at the head of column 3. This is the one bundred and forty-four
thousandth part the density of air. It is generally believed that the gases of the atwosphere distribute themselves in the long run, each as though the others were absent. Hence the new gas must extend a hun-
dred and forty-four thousand times as high as the heavy constituents of the air, to bring about the same proportionate reduction of pressure, even if gravitation the restraining influence of gravitation on the expan-
sion of the atmosphere diminishes as the square of the distance from the earth's center increases. It is eviion, that the new gas being present in the atmospher
must extend indefinitely into space without great los must extend indefinitely into space without great los o doubt that the new gas exists in the atmosphere though probably in very small proportion; perhap mrobable that it not only extends far beyond the atmosphere, but fills all celestial space at a very small pres-
sure. In recognition of this probability, I have provisionally named it aetherion or etherion ; meaning
"high in the heavens." Its symbol will naturally be I am aware that strong objections may well be raised to the hypothesis of an inter-planetary and intertellar atmosphere; but I can see no escape from the premises.
The estimated relative specific heat of etherion ap-
pears at the head of the fourth column, based again pears at the head of the fourth column, based again
on the assumed relative molecular velocity. In esti on the assumed relative molecular velocity. In esti
pating the specific heat. I have not made it inversely Dulong and Petit's law, giving a value ten thousand
times that of hydrogen; but have used the formula suggested by Prof. Risteen in his work on "Molecules gases shall vary with the number of degrees of free dom of their molecules. I have assumed as probably ng only three degrees of freedom.

Of course the values I have estimated for the mole-
cular weight, density, specific heat, and nolecula order of magnitudes we may expect to fo ind on fate the investigation; and it must not be forgotten that they
are based on two assumptions : first, that the heat conductivity of etherion is one huudred times that
of hydrogen; and second, that the ratio of heat conductivity and mean nolecular velocity is the same value here assigned to it. If so, the real values of its value here assigned to it. If so, the real values of its here given. The second assumption, while by no mation" to the relation between heat conductivity
and molecular velocity in gases. There is some evidence that etherion is a mixture of at least $t$ wo different gases. In the course of my ex-
periments I have met with a great many specimens,
obtained in various ways from various sources; but always mixed with a very large excess of other gases.
Some specimens were almost wholly absorbed by the phosphorus pentoxide at first userl for desiccation. being very rapid at first, but in an hour or two d wind-
ling to nothing and leaves a residue of gases permanently showing, by their heat conductivity, the presence
of a very considerable amount of etherion. Soda lime absorbed etherion, but much less freely than phos-
phorus pentoxide, and gave it up again on heating. The gas thus recovered was but little if at all affected In one experiment the

of pulverized window-glass, evolved from ten ounces hoda lime and then over fresh phosphorus pentoxide. Not a trace of etherion remained. The same result already referred to was used as the source of ethe-
rion. I will venture the conjecture that etherion will b orming one or more periodic groups of new eleproves true, I purpose to retain the present name for the lightest one.
The transmission of radiant energy through space
has always been to me a fascinating phenomenon, and I have indulged in much speculation concerning the it is effected. The remarkable properties assigned to
the ether from time to time in order to account for ob. served phenomena have excited my/keen interest; simpler explanation of the mechanism involved will
he found. To me a less strain of the imagination is required in the assumption that, instead of a continu-
o:s medium, gaseous molecules of some kind, endowed perhaps with a mode or modes of motion at present unknown to us, are the agent of transmission; a gas so subtle and lexisting everywhere in such small
quantity that it has escaped detection.
Perhaps the molecular hypothesis of the ether ha proved so attractive to me because it supports the vape that we may some time compass the perfect Such a vacuum would be opaque to light, and gravitative attraction could not, I believe,'act through rit. I
might afford alnew point of view from which to study the profound mystery of gravitation: an outsid
point.
The late De Volson Wood (Phil. Mag., Nov.. 1885)
considered the question of a gaseous ether mathe considered the question of a gaseous ether mathe-
matically, and deduced certain necessary properties of the hypothetical yas; chief among which were ex
ceedingly suall density and exceedingly high specific
heat Possibly we are about to find a gas which will ulfill the required conditions. It way be etherion, $c$ its lightest constituent if it turns out to be a mix
ture. I venture to express the hope that etherion will at least account for some phenomena at present attriUn account of the presumably extreme smallness of

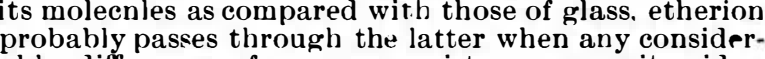
condensed or compressed in glass as before indicated. and may evaporate on the side of lower pressure, and
be absorbed on the side of higher pressure, after the manner of hydrogen in passing through palladium. n my own experiments the heat transnission ascribed to the ether way be due to the presence of the new
gas inside the bulb. A stnall fraction of a millionth would be sufficient, and this might escape detection compression in the gage he head causing absorption by
che glass. Again, etherion nust always be present to ome extent in all "vacuum tubes" (as well as in my evolution from glass; and may be the medium of proand air.

\author{
OSMIUM.
} he bluish white metal with violet luster, which $\mathrm{Mr}$.
Smithson Tennant discovered ninety years ago in the Smithson Tennant discovered ninety years ago in the
residue from dissolved platinum ores. This metal has lead 11'367, iron 7.79, and lithium, the lightest, only mium is likewise the most infusible of metals. It which makes platinum and iridium run like water. has of late employed successively in the manufacture of diamonds. Under this fierce heat the rare unetal
ruthenium, which used to be deemed all but infusible, readily melts. But osmium remains refractory, there his account osmium cannot be prepared in sufficient alloy with iridium, which'defies the acids, is of sone ip gold pens better having been found wherewith to for the bearings of the marinagnetic, its employment
vocated.-Popular Science News.

ALUMINUM CROWNS. *

A FEW weeks after making my first investment in office with a number of decayed teeth, which he wished me to save if possible. One, a lower second molar,
was in very bad shape for holding fillings, and I sugwith to him that it would be a good tooth to crow with a gold cap. But the idea of placing so much to his ideas. Here, thought I, is a chance to try one aluminum caps, and, setting a figure upon it which thought he would be willing to stand, I told him that
I here had something which would be just what he color, keeping qualities, durability, etc., he consented this material was even more easily manipulated than a
gold cap, on account of the pliability of the metal.
After shaping and fitting the cap and tooth to each other, I allowed the patient to bite, at first gently, then doing nearly all the swaging of the cusps and intervening depressions himself by the act of occlusion. place, and are of nearly uniform thickness in every usually do. Hence the ease with which the occlusion lators; in fact, the whole operation occupied but a lit-
tle inore time than it takes to make a good amalgam filling. This was nearly three years ago; since then I have first week of this present month. Everything was in good condition; the occlusion perfect, the color the praise for the work you so often hear of a well fitting
gold cap : "Doctor, that is the best tooth in my Case No. 2 was not so fortunate in its results. The patient, a young lady, presented a lower third molar,
which had been filled and refilled, and was then brok-
en down nearly to the gum margin. She thought the best thing for the tooth was extraction. But I sug-
gested placing an aluuninum cap, which would be much ess expensive than gold, and seeing what the result
would be. So that course was pursued. In this case I had to build up nearly all of the crown, which I did cap with cement and then pressing it to place, after having secured a good fit and articulation. The pulp
was alive, and responded for few moments to the action of the cement, as a healthy pulp nearly always does.
This crown did good service for nearly a year, when This crown did good service for nearly a year, when a
small break occurred in the margin of the grinding surface, caused by biting too forcibly upon a hard kernel of popcorn. The break seemed trivial and very what ignorance and thoughtlessness, combined with a the opening with an engine bur, made a nice undercut though the passage oressure exists on opposite sides,

THE heaviest thing in the world is the metal osmium
he bluish white metal with violet luster, which $\mathrm{Mr}$. y high the excessive-

By A. E. Preston, D.D.S., Delevan, N. Y. After a few questions concerning the metal, its that I should put it in place I at once proceeded
exactly as I would with a gold cap, and found that part, the same as swaged gold caps, and do not have
built up or filled in cusps, as our homemade crowns 
amalgam; then dismissed my patient, telling her that $\mid$ the usual methods. The whole of the materials re- $\mid$ double pulleys placed at the upper ends of the side

the crown was now as good as new. In the afternoon she returned bearing in her hand a crumpled, rotten, distorted piece of metal, which had no resemblance to my handsom aluminum cap; but
such it nevertheless was. This was her story : After eating dinner she sat down to talk with her husband, and remarked that that crown seemed loose. "She placed her fingers in her mouth. and saying. "It is ped it, exclaiming. "Oh, it's hot! It burned me." and at the same time picking the cap from the carpet and at the same time picking the cap from the carper,
He also dropped it, saying, "Gee whiz! It is hot. It' a wonder it didn't burn your mouth." That's just what I said also when she told me the story.
Now, one thing I want to know is, why didn't it burn her mouth? Were the fluids of the mouth keeping it cool, or was it waiting for a contact with the atmosphere to start up the heat action? I learned one
thing that I ought to have known before; that is, that mercury will unite with aluminum just as it will with silver or gold, and that amalgam should not be used to patch aluminum caps. I don't suppose any other that is why so few of them are ever brought to our attention. We prefer to tell of some of the brilliant successes we have had with some new appliance, or some intricate operation performed that has brough the little things of daily office practice, when remembered, help to make our work more successful.
I replaced the destroyed aluminum cap by another of I replaced the destroyed aluminum cap by another of
the same material, upon the good, solid cement crown, the same material, upon the good, solid cement crown,
which was well preserved, and it is doing good service which was well preserved, and it is doing good service
to-day. to-day. experience with aluminum caps has been very rightly placed. I would not use them when I could use gold just as well, yet I believe they are worthy of a
place in crown work.

\section{SETTING BACK BULGES IN FURNACES} BY a BOLLER INSPECTOR.

THE usual method of setting back bulges in furnace is to apply a fire below, and then to force the fire by a been sufficiently heated the fire is withdrawn, and a jack applied to force back the bulges. This nust be quickly done, or else the bulge will have cooled down
so much that it cannot be forced back. In the limited roou for working, it is always an awkward and un pleasant jub. In removing the fire it of ten gets spilled bulge has also to be cleared out of the furnace the during the time this is being done the heated part is rapidly cooling. For rebeating the fire has again to be packed up against the bulge, and the cycle of operations repeated till the furnace is sufficiently restored to
shape. The whole of the work has invariably to be shape. The whole of the work has invariably to be
performed at the place where the boiler is worked, with performed at the place where the boiler is worked, with
what appliances can be most conveniently found, of ten by workinen who have had no experience with this class of work.
The writer designed the method shown on annexe connected by joints, $E$ and $F$, so that there is ther are with this job, and he believes it is an improventent on and lowered, as need may be, by the ropes, $G$, and the A places, with little trouble. plugged up, the upper part inside the bucket perforwith fireclay, ar several holes, and the bucket lined cipped on near the other end of the pipe, that end be ing connected, preferably by a hose pipe, with the
rivet-fire bellows. The fire is kindled and got properly started before inserting in the furnace. A suitable tube. The fire can be quickly placed in position un der the bulge, and by grasping the cross handle and quired. It can be quickly withdrawn, and laid down

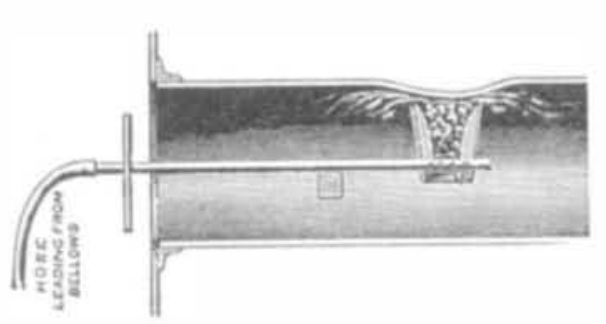

way, and the jack applied to the bulge with the miniA 15 to 20 ton hydraulic jack is the best to use. small piece of $1 / 2$ inch or $5 / 8$ inch plate, heated to red and marking the furnace plate. A larger plate, ben jack to distribute the stress. If any packing is required to bring the jack against the bulge, a single be used. The bulge should be pressed back by the jack; at the same time a little judicious flogging on

After the furnace has been restored as near as pracback should be heated to a dull red to remove any local stresses that may have been set up in the plates.
As the fire can be easily shifted about to any desire part of the furnace, this can be easily done.-EngOLD METHODS OF CROSSING RIVERS BY MODEL OF' A PONTOON OR PORTABLE BOAT FOR ROW RIVER. the usual methods. The whole of the materials re-
quired can generally be got, even in the most out-of-the-

witted to the bottom of a wrought-iron bucket in abeter, is The end, which passes a short distance through, being put on the top of the jack to prevent it from cooling block of good hard wood of suitable thickness should

THIs machine can be used very advantageously, proprir boats into the water, even in broad daylight. troops is not more than from 40 to 45 feet in width. It of the besiegers. consists of a pontoon, $A$, which is provided with three Nevertheless, it is better to take advantage of the in that the enemy can then throw fewer obstacles in The portion of this bridge marked $C$, which is that of the center, may, by means of the pivot, $I$, be put the pontoon is on the move, but is placed crosswise portions, $B$ and $D$ fold upon bridge. The two other upon the one marked $C$; and, to this effect, the joints portion marked $B$. All the pieces of this bridge and
portion those that may be added thereto in order to strengthen it as well as the pontoon or boat. $A$, are loaded upon a in the figure, and upon which the machine can be easily carried from one place to another. When it is desired to make use of it, all that is to be done is to pute the boat, $A$, into the water, anchor it in the midand passing the ropes, $G$, over the pulleys posts, $H$, the portions, $B$ and $D$, and thus form a bridge of the

Thus one maneuver is effected in a very short time. march and the construction of a bridge, and allow troops to pass over it for surprising the enemy, or for

DEL OF' A PONTOON FOR CROSSIN THE MOAT OF WITH WHICH IT IS FILLED IS STILL.

In the siege of places where the situation does not permit of drawing off the water of the moats or of conveniently filling the latter in there may be used able to cross the moat in order to place a mine or to ascend to an assault.

The boats or pontoons that I propose for this pur and, during carriage, are united in pairs, one on top When it is desired to use them, they are assembled at the head of the moat, and afterward, in measure a each other end to end and pushed to the side of the The first boats placed in the water are pushed by the following ones in succession until they form a bridge of the necessary length over the moat.
The wheels with which these boats are provided serve not only for moving them from one place to sired to put them in water or to remove them therefrom, provided, noreover, that a glacis be formed at ent to the moat-a And, this glacis once formed, the laborers employed in the construction of the bridge will be able to work in the moat under cover from the fire of the besieged. ing the night, one is in a state to surprise the enemy

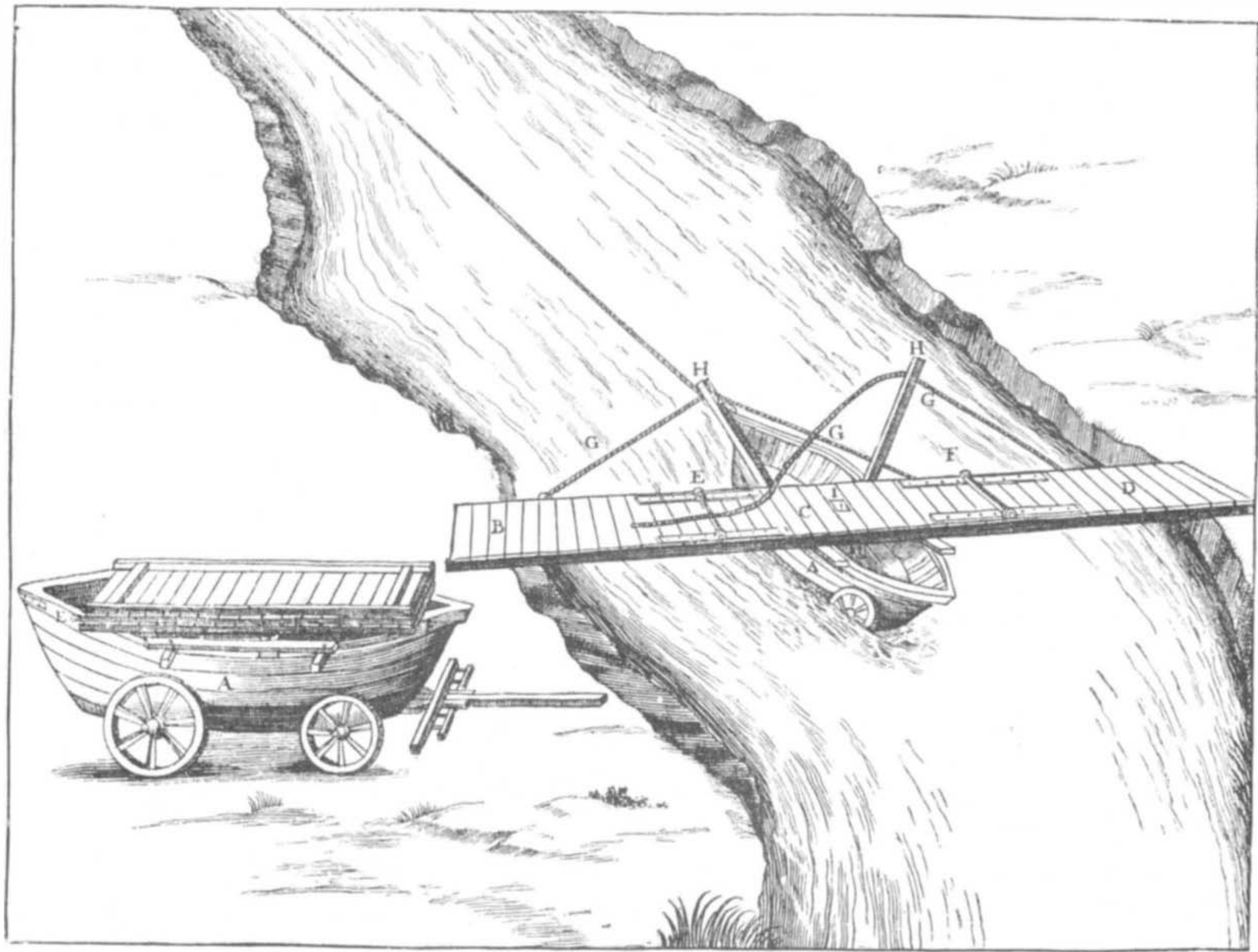

MODEL OF A PON'TOON OR PORTABLE BOAT FOR PROMPTLY THROWING A BRIDGE ACROSS A NARROW RIVER. 